\title{
Optimum bit rate for image transmission over underwater acoustic channel
}

\author{
Hamada Esmaiel, Danchi Jiang \\ School of Engineering, University of Tasmania, Hobart, Australia
}

Email address:

Hamada.Esmaiel@utas.edu.au (H. Esmaiel), Danchi.Jiang@utas.edu.au (D. Jiang)

\section{To cite this article:}

Hamada Esmaiel, Danchi Jiang. Optimum Bit Rate for Image Transmission over Underwater Acoustic Channel. Journal of Electrical and Electronic Engineering. Vol. 2, No. 4, 2014, pp. 64-74. doi: 10.11648/j.jeee.20140204.12

\begin{abstract}
In this paper, image transmission in underwater channels is considered. The images are encoded with forward error correction using an unequal error protection technique together with the Reed-Solomon codes and dynamic bit-rate allocation before transmitted. This paper proposes a novel rate allocation scheme for efficient image bit stream transmission in underwater acoustic channels with optimum bit rates. The optimality is achieved in the sense that the comprehensive peak signal-to-noise ratio of the image transmission is maximized under channel bit rate and bit error rate constraints. Based on a modified set partitioning in hierarchical trees (M-SPIHT) image coder, four different flocks of bit-streams based on their significance levels are generated. According to their significance levels, the blocks of the significant bits, the sign bits, the set bits and the refinement bits are transmitted with different protection levels, so as to reduce the total distortion of received image. In addition to the careful selection of each component and intuitive justification in the detailed system design, simulation results have also been included. It is demonstrated that the proposed scheme outperforms the equal error protection for image transmission in underwater channels, significantly improves the peak signals-to-noise ratio (PSNR) performance in comparison to existing coding schemes.
\end{abstract}

Keywords: Rate Allocation, Reed Solomon Coder, SPIHT Coding, Underwater Acoustic Channel, Unequal Error Protection (UEP)

\section{Introduction}

Wireless underwater acoustic (UWA) communication systems are sensitive to numerous of scientific and civilian tasks in the water of the ocean, such as observation and reconnaissance for the oceans, undersea rescue, and emergency response to undersea disasters, etc. Modern high data rate communications for image transmission are desirable for efficient underwater expeditions. As the attenuation is significant in cases where electromagnetic waves are used in the wireless communication system for the salt water, acoustic waves are the viable wave to carry the information in this case. However, existing acoustic communication technologies currently available can only support a limited data rate communication due to limited channel bandwidth available in addition to multipath spreading in the UWA channel [1-3]. In fact, as the special physical characteristic of the underwater channel, wave propagation is significantly affected by temperature, salinity of water, activity of hydrogen ions $(\mathrm{pH})$ of water, depth of the water column or pressure and surface/bottom roughness [4].
The high error rates, significant path loss, and low channel capacity are typical features of underwater channels and that make the task of high speed data transmission even more challenging than that with terrestrial radio links [5].

One of challenges of image transmission in an UWA channel is a large amount of long burst errors. The underwater acoustic channel fading can causes significant long burst errors and sporadic failure, for that the image transmission is far more challenging than that in stationary and more predictable wired or wireless environment. With a conventional image coder, image transmission signals over UWA channel can be significantly dispersed and distorted. For that sophisticated coding techniques will be needed. However, that may increase the overhead in spectrum use. In [6] is known that effective transmission over UWA channels is dependent on two factors: (1) efficient compression techniques and (2) well-organized bandwidth. In this paper, we attempt on both aspects where efficient data compression will be achieved by using effective wavelet based progressive 
image compression technique called set partitioning in hierarchical trees (SPIHT) [7], aiming to decrease the mean-squared error (MSE) between encoded and decoded image. The bandwidth efficiency is achieved using unequal error protection optimization technique formulated by taking the particular channel feature into account at the transmitter $[8,9]$.

In this paper, a system will be designed to minimize the transmission error of image bit-streams under a total broadcasting rate constraint. Unequal error protection (UEP) [10] will be proposed for high quality image transmission. UEP uses four different bit stream groups outputed from a modified SPIHT [7] source code. The expected respective contributions of each group at the overall peak signal-to-noise ratio (PSNR) in the decoded image will be estimated based on the corresponding level of protection in each group determined. The reason that SPIHT is selected to use for underwater acoustic (UWA) channel because it is inherently designed for image encoding, and the associated redundancy can be managed conveniently for communications and its capability to adapt to actual channel constraints [11]. A Reed Solomon (R-S) channel coder will be adopted in this paper to enhance error correction capability. Authors in [11] have used a fixed UEP technique where more important data is protected with more redundancy and the less important data is protected by limited redundancy. The redundancy amount can be fixed for each packet. In this paper, we try to develop an adaptive UEP technique based on the SPIHT bit stream sensitivity, the noise level of the underwater acoustic channel, and its effects on the overall channel distortion.

This paper is organized as follows: The set partitioning in hierarchical trees SPIHT with unequal error protection algorithm, together with underwater channel will be introduced in Section 2 as preliminaries. The proposed coding algorithm for underwater acoustic channel to reduce the channel distortion will be developed in Section 3. An intuitive explanation behind the various aspects of the proposed UWA communication system will be given in Section 4 . The proposed system will be simulated under a set of typical underwater channel parameters and the illustrative outcomes are given in Section 5. Finally, the conclusion and summary of the major techniques and contributions of this paper will be included in Section 6 .

\section{SPIHT Coder with UEP and Underwater Acoustic Channel}

Sophisticated image compression techniques based on wavelet algorithms have been reported for the underwater transmission. Please see $[5,10,11]$ for some examples. In these works, SPIHT coder has been demonstrated of advantages in many aspects including advanced bit error ratio, continuous-tone with a high compression ratio, lossy and lossless compression, and progressive processing. SPIHT was originally introduced by Said and Pearlman [7]. It improves PSNR for certain compression ratios for a broad assortment of images compression coders [12]. As such, it has been widely used in image application, and set as an essential performance benchmark for the evaluation of new image coding techniques. These features make the SPIHT compression technique a reasonable choice for realistic underwater transmissions. In this line, a simple source compression scheme incorporated with SPIHT source coding scheme has been investigated earlier in [5], for underwater transmissions. The encoded bitstream was divided into consecutive chunks before transmitting over underwater links. The expected attenuation of UWA channel is one of the major channel challenges. In addition, restrictions due to distance, depth, frequency, temperature and acidity to salinity of the UWA channel make the communication channel to be dynamic and fast time varying, which significantly affects communication system behavior. In order to make further improvements, it is necessary to apply appropriate underwater channel models to lay a foundation for the discussions it in this article, which will be detailed in this section.

\subsection{SPIHT Coded Output Bit Stream Classification}

Set partitioning in hierarchical trees (SPIHT) is an algorithm established based on the hierarchical partitioning of the wavelet coefficients set and organize it into a spatial orientation trees, where all sub-bands coefficients are included in the corresponding branches to identify their particular position in the image [7, 13, 14]. All insignificant coefficients are sequentially encoded together as an insignificant symbol set. An appropriately defined partitioning rule can be utilized to further divide this insignificant set to progresivelly extract the significant coefficients iteratively. SPHIT coder is considered the best available coder due to progressive rate control and transmission, as well as coding process simplicity, etc. [7]. In particular, after image decomposition with 9/7 tap wavelets by the method reported by Antonini et al [15], the general SPHIT coding algorithm encodes image by splitting the decomposed image into considerable sections on the basis of the significance classification function as:

$$
S_{n}(\Gamma)=\left\{\begin{array}{cc}
1, & \text { if } \quad \max _{(i, j) \Gamma \Gamma}\left\{|Y(i, j)| \geq 2^{n}\right\} \\
0, & \text { otherwise }
\end{array},\right.
$$

where $Y(i, j)$ represent wavelet coefficient at the pixel $(i, j)$ for $n$ bit plane. The algorithm works on the basis of two tiers of loops, the sorting loop and the refinement loop. To implement SPHIT coder three related groups [16] are obtained: first index of insignificant pixels, the index of significant pixels, and the index of insignificant sets. A quantization process is applied to sort the wavelet coefficients of these different groups using a different quantization level. On the basis that each subset is divided into different groups ordered according to their significance levels, the most important wavelets coefficients in the image 
are encoded with a bit-stream to realize the best encoding quality than the previous schemes [17]. While the algorithm initially generates a list of insignificant pixels (LIP), then evaluates its element's significance versus updated threshold value and encodes their relevance by 0 or 1 . For the significant coefficients, the signs of the coefficients are encoded together with the corresponding pixel. Then the encoded pixels are sent to a list of significant pixels (LSP) group. After checking the list of insignificant sets (LIS), the threshold for the significant set coefficients will be updated. The particular tree/set significant bits are classified into children or grandchildren based on the updated significance thresholds. Otherwise, for the insignificant set it is represented as a zero-tree in the encoded bits in the output bit streams. At the end of each iteration, the classification process determines the refinement bits. Its output from the SPIHT algorithm at the updated bit significance pixels is moved to the LSP at each threshold update, leading to the refinement of significant pixels with rate reducing error. The threshold is incremented with two steps until the output bit-stream ends.

In this paper, the sorting pass will be performed on separate four lists rather than three: LIS, LIP, LSP, and the roster for refinement path. The LSP and LIP consist the nodes indicate the single pixels while the descendant nodes are represented by the LIS. The highest coefficients can be represented by the number of bits in the following formula:

$$
n_{\max }=\log _{2}\left(\max _{\{i, j\}}\{|Y(i, j)|\}\right),
$$

which is similar to that in [7]. While screening image pixels, classified LIP of pixels can be obtained based on the significance degree using Equation (1). The resultant $S_{n}(\Gamma)$ is then forwarded to the coded bits. All those significance pixels will be sent to the LSP group with their sign bits sent to the output stream. Similarly, for sets in LIS they will be tested and sent to LSP or LIP according to their significance levels. For refinement loops, the $n^{\text {th }}$ generality significant bit of the coefficients in the LSP is used. By iteratively going through all $n$ that is decreased by one, the desired rate can be obtained until every LSP node is processed.

\subsection{Underwater Acoustic Channel}

The UWA channel can be highly distorted. One of the most challenging issues in it is the significant propagation delay, in addition to the high attenuation level with low propagation velocity. All of this makes the UWA channel to be one of the most challenging environments for wireless communication [18]. Many models have been developed to predict the UWA channel attenuation, which is dependent on several factors of UWA channel. For example, the carrier frequency, communication distance, acoustic sensor depth, temperature and acidity to salinity of the UWA channel [19].

\subsubsection{Propagation Loss}

Before The communication distance is inversely proportional to the power of acoustic signal in UWA channel. UWA channel is influenced by physical channel characteristics such as boric acid, magnesium sulphate, geometric spreading, and particle motion. UWA channel propagation loss is affected by three parameters characterizing of the geometrical spreading, attenuation and the abnormality of spreading. Other factors cannot be easily modeled. Accordingly, the expected attenuation of the propagated signal, in $\mathrm{dB}$, with respect to the transferred range $l$ and a carrier frequency signal $f$ can be approximated as $[4,8,20,21]$ :

$$
10 \cdot \log A(l, f)=10 \cdot k \cdot \log l+10 \cdot l \cdot \log \alpha
$$

where $k$ is the geometrical UWA channel spreading factor. Its value is usually between 1 and 2 . In this paper, we consider value $k=1.5$ for illustrative purpose. $\alpha$ is the absorption coefficient in $\mathrm{dB} / \mathrm{km}$.

To characterize UWA multipath effects, we consider the $p$-th path as a generic case. Its effect with a delay $t_{p}$ and a mean magnitude gain $\alpha_{p}$ can be estimated. The UWA channel multipath magnitude and tab delay values are a function in the length of the communication path $l_{p}$. The UWA channel path gain is given by $\alpha_{p}=\Gamma_{p} / \sqrt{A\left(l_{p}, f_{c}\right)}$. Assuming that the path gain is remains the same value over each path, $\Gamma_{p}=(1 / \sqrt{2})^{r_{p}} . r_{p}$ is an integer associated with the number of reflected paths. i. e., $r_{p}=0,1,3,5,7$, for the main path, first path, second path, ..., respectively. The propagation loss in this path can be obtained by Equation (3), $A\left(l_{p}, f_{c}\right)$, calculated by using the following Equation:

$$
A\left(l_{p}, f_{c}\right)=l_{p}^{k}\left[\alpha\left(f_{c}\right)\right]^{l_{p}},
$$

The path delay $t_{p}$ is given by $t_{p}=l_{p} / c$ (the speed of sound in the water is $c=1500 \mathrm{~m} / \mathrm{s}$ ) [22]. Path length $l_{p}$ can be obtained based on geometry plane model as in [22].

\subsubsection{Ambient Noise Model}

The UWA channel ambient noise can have a continuously changing power spectral density (p.s.d.) [4]. The significant factors on the level of the UWA channel ambient noises are the shipping, turbulence, wind waves and thermal noise. Each factor has an ambient noise p.s.d. in $\mathrm{dB}$ re $\mu \mathrm{Pa}$ per $\mathrm{Hz}$. It can be approximated using the formulae (5), (6), (7), (8) shown below, respectively [4]:

$$
\begin{aligned}
& 10 \log N_{s}(f)= 40+20(s-0.5)+26 \log f-60 \log (f+0.03), \\
& 10 \log N_{t}(f)=17-30 \log f \\
& 10 \log N_{w}(f)=50+7.5 \sqrt{\omega}+20 \log f-40 \log (f+0.4), \\
& 10 \log N_{t h}(f)=-15+20 \log f
\end{aligned}
$$

Ambient noises are functions of the carrier frequency. The overall effect of the ambient noise can be formulated as [4]: 


$$
N(f)=N_{t}(f)+N_{s}(f)+N_{w}+N_{t h}(f) .
$$

UWA channel performance is dependent on the composite ambient noise and channel multipath attenuation.

\subsubsection{Signal-to-Noise Ratio}

UWA channel signal-to-noise ratio (SNR) is a function of attenuation $A(l, f)$ as well as the ambient noise $N(f)$ [23]. The SNR of the UWA channel at the receiver can be calculated as:

$$
\operatorname{SNR}(l, f)=\frac{P}{A(l, f) N(f) \Delta f} .
$$

Notice that SNR in Equation (10) is calculated in $\mu \mathrm{Pa}$ per $\mathrm{Hz}$. The expected SNR is a function in communion range $l$, the signal carrier frequency $f$, transmitted signal power $P$ and expected receiver noise bandwidth $\Delta f$. As such, the expected UWA channel SNR can be maximized by using an optimal carrier frequency. The SNR for signal transmission can be predicted using the knowledge of the transmission range and the carrier frequency.

\subsubsection{Doppler Shift in UWA Channel Multipath}

The Doppler shift is one of the most challenging factors in the fast time varying channel. A Doppler shift in the UWA channel is different from wireless channels. It is not only a relevant to the frequency shift, but also to the low propagation speed. In low propagation speed a small receiver movement produces an impact Doppler shift [24]. The phase of the Doppler shift $\varphi_{d}$ depends on the receiver motion velocity $v$ and ratio of the modulated symbol rate $S_{R}=1 / T$ to carrier frequency $f_{c}$ [25]. The UWA channel Doppler shift is given as:

$$
\varphi=-2 \pi f_{c} T \frac{\Delta}{1-\Delta}=-2 \pi \frac{f_{c}}{S_{R}} \frac{v}{c-v}
$$

\section{Coding Operation over Underwater Acoustic Channel}

Advanced underwater acoustic with error correction coding is proposed to long range underwater acoustic communication [26]. In that paper, $\mathrm{R}-\mathrm{S}$ block channel code is proposed with adaptive unequal error protection. It has also been reported in [27] that UEP enhance significantly the quality of the transmission operation as well as minimize the transmitted rate in highly distorted channels. Albanese et al. succeessfully adopted the UEP to transmit packets over networks in their priority encoding transmission (PET). Albanese technique is based on optimized rate as addressed in $[28,29]$. In this paper, the UEP technique will be applied from another perspective. A modified SPIHT (M-SPIHT) source encoder will be proposed to generate four various types of bit-streams based on encoded bits significance in decoded image quality. M-SPIHT output bit-streams are obtained based on the contribution of each bit-steam in the
PSNR of the received image. Then the protection level of each bit-stream will be adjusted adaptively by using $\mathrm{R}-\mathrm{S}$ channel code.

\subsection{Problem Formulation}

M-SPIHT is proposed to classify bits to four different types of groups based on their sensitivity levels and their contributions in the reconstructed image PSNR.

Bit error sensitivity (BES) analysis can be carried out assuming the image is encoded using an M-SPIHT coder. For analysis purposes, assume the encoded image is corrupted progressively bit by bit orderliness from beginning until the output bit-stream ends. For oblique bits, the decoded image MSE is calculated. The oblique bit is repaired first, then the same procedure is applied to the next bit. The proposed M-SPHI produces four major types of bit-steams with different levels of sensitivity. They can be given in order as: (1) the significance bit (2) the sign bits; (3) set bits to determine if setting significantly, (4) refinement bits. In Figure 1, it can be observed that the bit's groups are ordered by their significances from highest to lowest for a $256 * 256 * 8$ Lena image and it's bits can be ordered as: significance bits $>$ sign bits $>$ set bits $>$ refinement bits. The four different bit-streams have different significant levels in the received image PSNR. Channel coder overhead information is added for each transmitted packet adaptively by an optimization algorithm. The optimization algorithm is based on the length of the packet, the expected UWA channel SNR and the expected MSE is decreasing in each packet when a packet is received correctly. Depending on the rate allocation vectors and $\mathrm{R}-\mathrm{S}$ overhead length the bit-stream is transmitted.

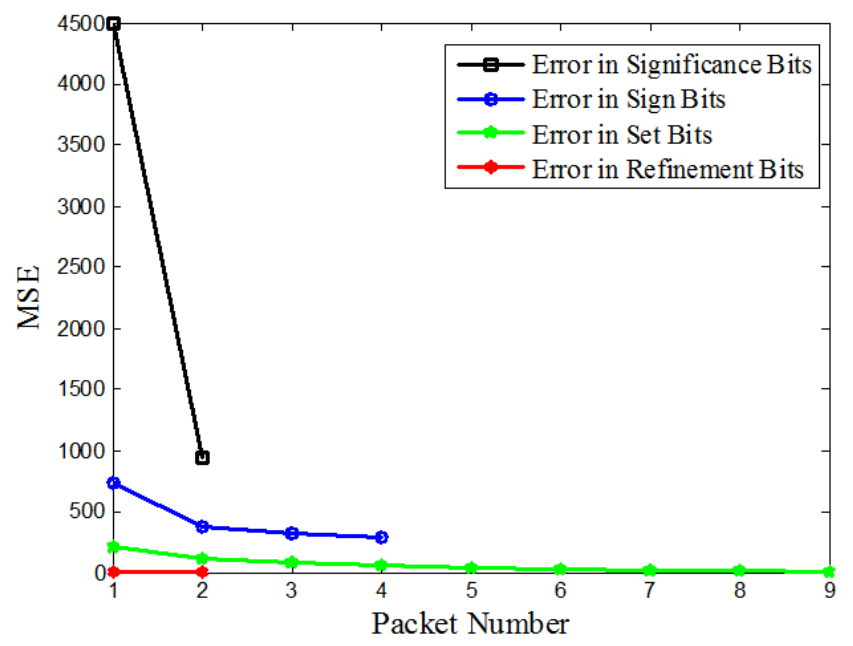

Figure 1. M-SPIHT expected packet sensitivity to error.

The forward error correction (FEC) will be using a R-S coding for data transmitted over UWA channel. Assuming the M-SPHIT encode image and its output splits into a series of packets, if $\Delta D_{i} \geq 0$ is the predict MSE reduction in the decoded $i^{\text {th }}$ packet, the total deformation in total received packets can be formulated as: 


$$
D(l)=D_{o}-\sum_{i=1}^{l} P_{i} \Delta D_{i}
$$

Here, $D_{o}$ is the MSE expected for transmitting packet without any overhead information. $P_{i}$ is the probability that $i^{\text {th }}$ packet correctly received over the channel, and $l$ is the total transmitted packet number. $P_{i}$ is the probability of correctly received packet over the UWA channel. It is a function in the transmission data rate and it can be written as:

$$
P_{i}=\prod_{j=1}^{i} Q_{j}\left(r_{j}\right)
$$

where $Q_{j}\left(r_{j}\right)$ is the probability of receiving the $j^{\text {th }}$ layer correctly at receiver side when it is set at a rate of $r_{j}$. Applying Equation (13) to Equation (12) yields:

$$
D(l)=D_{o}-\sum_{i=1}^{l}\left(\prod_{j=1}^{i} Q_{j}\left(r_{j}\right)\right) \Delta D_{i} .
$$

Optimal allocation rate $r_{j}$ is the amount of total bits minimizing the received packet distortion based on the Equation (14) at a constant transmission rate. The minimal distortion problem can be formulated generally as follows:

$$
\min _{r} D(r) \quad \text { Subject to } \sum_{j=1}^{l} r_{j} \leq R,
$$

where $R$ is the total transmission rate in the equal error protection system (EEP) and it must not exceed the channel capacity.

\subsection{UWA Channel Reed Solomon Coder}

Reed Solomon is a type of forward error correcting codes. It is a systematic linear block code $[9,30]$. Each code block is organized into m-bit data-words units with a specific length. Its length is usually between 3 and 8 bits. For example, with 8 bit code length all probable data-words are suitable for coding. Each systematically generated code-word is composed of the original version of the encoded data-word in addition to the "parity" symbols to be subjoined it. Symbol error probability (SEP) of $\mathrm{R}-\mathrm{S}$ over communication channel is $P_{E}$. For hard input $\mathrm{R}-\mathrm{S}$ decoder and assuming the independence of the errors to the channel SEP and $P_{E}$ is a function in symbol-error correcting capability $p_{e}$ the maximum number of correctable errors $t$ can be written as follows [31]:

$$
P_{E} \approx \frac{1}{2^{m}-1} \sum_{s=t+1}^{2^{m}-1} s\left(\begin{array}{c}
2^{m}-1 \\
s
\end{array}\right) p_{e}^{s}\left(1-p_{e}\right)^{2^{m}-1-s},
$$

where $m$ is the number of bits/symbol. The maximum number of correctable errors $t$ for a $(\mathrm{N}, \mathrm{K}) \mathrm{R}-\mathrm{S}$ code can be calculated as $t=0.5(n-k)$.
Shallow water UWA noise is neither Gaussian nor white distributed [32-34]. In [35] the authors reported a model of probability density function (pdf) for the underwater acoustic noise issue based on real measurements at sea. An analytical expression is given for the UWA channel SEP in case of using BPSK transmission. For binary signaling, the related likelihood functions are obtained and an SEP expression is obtained. The SEP of the binary UWA noise channel can be estimated as a Student's $t$ pdf and it is expressed by [35]:

$$
P_{e}=k \sqrt{2 E_{b} / N_{o}} \int_{0}^{\infty}\left[\left(4 E_{b} / N_{o}\right)(x+1)^{2}+5\right]^{-2.75} d x
$$

Here, $x$ is a vector of amplitude noise $M$ level. $E_{b} / N_{o}$ is the energy per bit to noise power (normalized SNR) and $k$ is an integer $k=1,2, \ldots \ldots, M$.

The noise average power spectral density is $N_{o}=\sigma^{2} / B$, where $B$ is the bandwidth and $B=1 / 2 T_{b}$. When the amplitude of the pulses is unitary, the noise variance $\sigma^{2}$ could be related to the SNR per bit $E_{b} / N_{o}$, and it will be:

$$
\sigma^{2}=\frac{1}{2 E_{b} / N_{o}}
$$

\subsection{Optimization Technique}

The output transmitted bit-stream can be split into different coding blocks. Each of these blocks includes $k$ source packets. Each block is encoded by $(\mathrm{N}, \mathrm{K}) \mathrm{R}-\mathrm{S}$ channel coder. In case of $(\mathrm{N}, \mathrm{K}) \mathrm{R}-\mathrm{S}$ channel coder $(\mathrm{N}-\mathrm{K})$ is the maximum overhead information required to protect each block. Then, these packets are transmitted over UWA channel. Let $P_{e}$ be the symbol error probability of the channel. Optimal rate allocation vector $r$ for Equation (15) can be obtained by using the Lagrange multipliers Equation as:

$$
J(r, \lambda)=D(r)+\lambda \sum_{i=1}^{l} r_{i}
$$

The correct receiving conditional probability at transmission rate $r_{j}$ of the $j^{\text {th }}$ source packet is $1-P_{E}\left(r_{j}\right)$. Then, Equation (13) becomes:

$$
P_{i}=\prod_{j=1}^{i} Q_{j}\left(r_{j}\right)=\prod_{j=1}^{i}\left(1-P_{E}\left(r_{j}\right)\right),
$$

Apply the Equation (14) and Equation (20) into equation (19) one obtains:

$$
J(r, \lambda)=D_{o}+\sum_{i=1}^{l}\left[\left(-\prod_{j=1}^{i} Q_{j}\left(r_{j}\right)\right) \Delta D_{i}+\lambda r_{i}\right]
$$

This function in Equation (21) can be optimized to determine the appropriate $r_{j}$. It is dependent on the set of distortion increments $\Delta D_{i}$, as well as the probability that 
the $j^{\text {th }}$ layer source packet is received correctly. There are many methods available to solve this optimization problem. It can be solved based on an alternating variables iterative method [36]. $J=\left(r_{1}, \ldots \ldots \ldots \ldots, r_{l}\right)$ in Equation (21) is the objective optimization function. It is used to minimize one variable each time. The objective optimization function is keeping other variables constant until the iterative process reach to the convergence. Let $r(0)$ is the initial rate allocation vector and $r(t)=\left(r_{1}(t), \ldots \ldots, r_{l}(t)\right)$ is obtained values at $t=1,2, \ldots$ as follows: for $x \in\left\{r_{1}, \ldots \ldots, r_{l}\right\}$ to be optimize at $t$ step this done in a round-robin way. In the next step, for $x=r_{i}$, following rate optimization is performed:

$$
\begin{gathered}
f(r)=r_{i}^{(t)}=\underset{D_{i}}{\operatorname{argmin}} J\left(r_{1}^{(t)}, \ldots \ldots, r_{l}^{(t)}\right)=\underset{r_{i}}{\operatorname{argmin}} \sum_{i=1}^{l}\left[\left(-\prod_{j=1}^{v} Q_{j}\left(r_{j}\right)\right) \Delta D_{i}+\lambda r_{i}\right] \\
D\left(r_{1}, r_{2}, r_{3}, \ldots \ldots \ldots ., r_{l}\right)=D_{o}-\sum_{i=1}^{l}\left(\prod_{j=1}^{i} Q_{j}\left(r_{j}\right)\right) \Delta D_{i}, \\
R\left(r_{1}, r_{2}, r_{3}, \ldots \ldots \ldots \ldots, r_{l}\right)=\sum_{i=1}^{l} r_{i},
\end{gathered}
$$

where $r$ is the overall transmission rate in the unequal error protection system (UEP) for each transmitted packet with total transmission rate $\sum_{j=1}^{l} r_{j} \leq R$.

$R$ is the maximal total transmission rate in the equal error protection (EEP) system.

The Lagrange multipliers method can also be used to find the point of minimum overall distortion $D$, across all transmission rates $R_{n}$ in the unequal error protection system
(UEP) with such maximal rate constraint. It leads to:

$$
\begin{aligned}
& \left.\frac{\partial}{\partial \vec{r}}\left(D+\lambda\left(R_{n}-R\right)\right)\right|_{\vec{r}=r^{*}}=0, \\
& \left.\frac{\partial}{\partial \vec{r}}\left\{D_{o}-\sum_{i=1}^{l}\left(\prod_{j=1}^{i} Q_{j}\left(r_{j}\right)\right) \Delta D_{i}+\lambda\left(\sum_{j=1}^{l} r_{j}-R\right)\right\}\right|_{\substack{\overrightarrow{\mathrm{r}_{\mathrm{k}}}=\overrightarrow{\mathrm{r}_{\mathrm{k}}^{*}} \\
.}}=0 .
\end{aligned}
$$

The gradient-descent type algorithms as a standard non-linear optimization procedure can also be used to minimization optimization problem for a fixed $\lambda$ [36].

\section{Proposed UWA Communication System}

Based on the considerations listed in Sections 1, 2, and 3, we propose a communication system scheme for UWA channels as shown in Figure 2. The major components are: (1) the M-SPIHT coder used to generate a four types of bitstream regards to its significance order; (2) data outputs which is split into a series of stream packets; (3) the predictable decrements in $\Delta D_{i}$ that can be approximately estimated based on the expected change in MSE; (4) the optimization algorithm to generate the optimum bit rate distribution of packets, which is adaptive to the optimization algorithm inputs, including packet length, expected decrease in distortion $\Delta D_{i}$ and channel SNR; (5) R-S encoder used the output rate allocation vectors to generate the transmitted bit-stream; (6) the receiving bit-stream to be decoded by using $\mathrm{R}-\mathrm{S}$ decoder the M-SPIHT decoder.

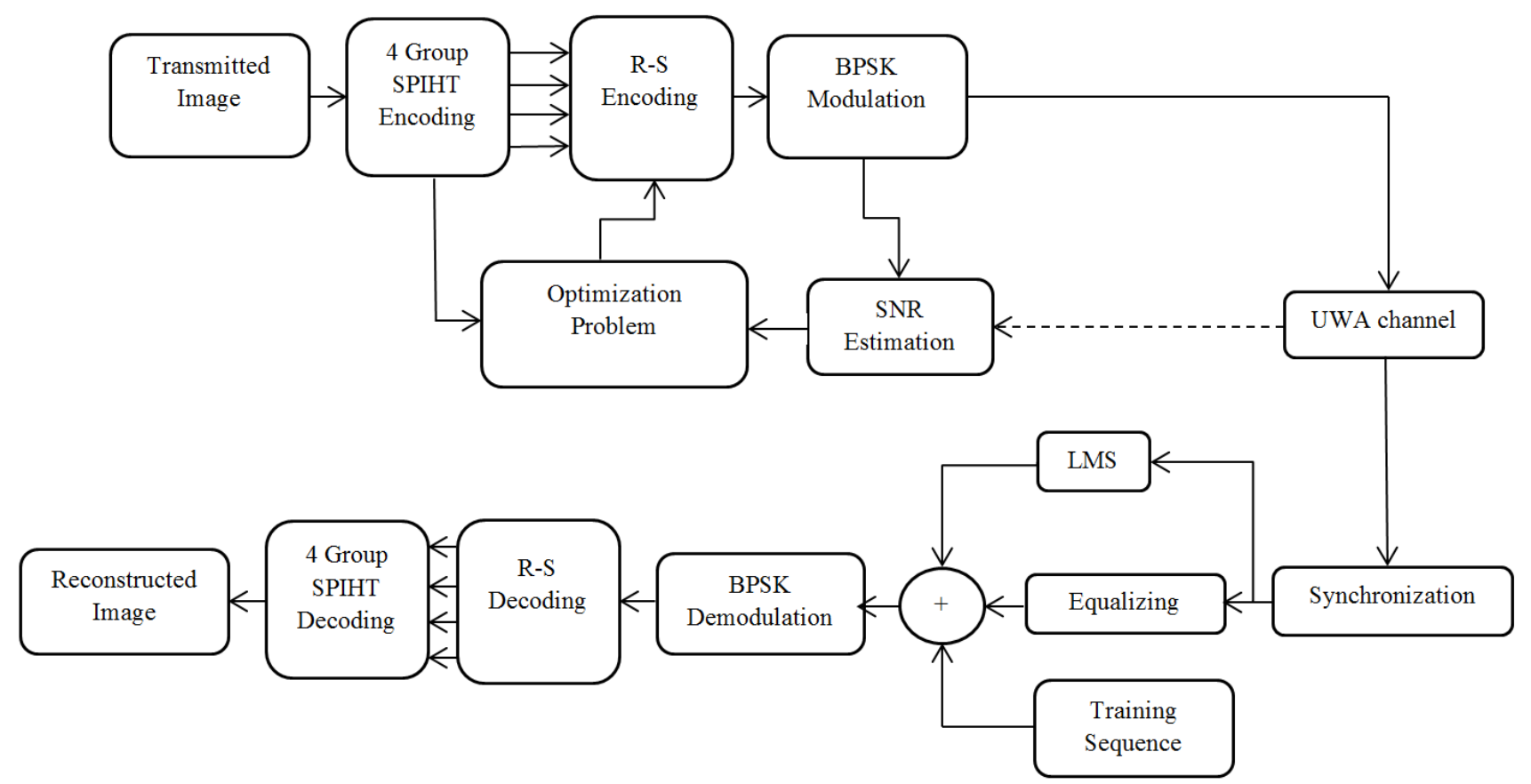

Figure 2. System configuration. 
An SNR estimation block is included in the diagram in Figure 2. In this paper, SNR is estimated using equation (10) which depends on the distance between the transmitter and receiver. The carrier frequency, signal transmission power and receiver noise bandwidth are all known at the transmitter. Another important parameter in the equation (10) is the ambient noise (considered as feedback signal) and it depends on physical channel characteristics. Equation (9) can be used to approximate the ambient noise. This block can also be integrated with other information source such as the bit-error rate estimation from network management protocols to facilitate direct feedback from the real time communication status to the optimal bit protection level allocation.

At the beginning, the SPIHT coder output bit-stream modification has been introduced. The proposed UWA communication system for image transmission is based on the sensitivity of each block of M-SPIHT output and its contribution in reconstructing image PSNR.

The BES can be evaluated with corrupted M-SPIHT encoder outputs bit-by-bit, starting from the first bit to the last one. For each corrupted bit, the MSE of original and the reconstructed image is calculated. For each iteration the last corrupted bit is corrected first. Then, the next bit is corrected and transmitted.

\section{Simulation Results}

\subsection{Simulation Setup}

In this section, the simulation results using proposed M-SPHIT coder algorithm for image transmission over the UWA channel will be presented. Communication system described in Section 4 is implemented. $256 * 256 * 8$ Lena image is used as the source image encoded using M-SPHIT with 0.5 bpp compression rates. The resultant BES is depicted in Figure 1. As shown in the Figure 1, the output is ordered starting from the highest significant bits (a first group of bits). We consider a link of $5 \mathrm{Km}$ in shallow water and take into account different wind speeds, while water depth is $40 \mathrm{~m}$, the relative velocity is set to be $8 \mathrm{~m} / \mathrm{s}$. The attenuation-noise (AN) factor is obtained as $8.6 \mathrm{KHz}$ as the optimal carrier frequency for our suggested underwater channel physical parameter according to Equation (10). Table 1 shows the calculation results of channel relative delays and magnitudes for each path. The wind causes significant waves, scatters the surface reflection, and gives these reflections a Doppler shift. For that decision-feedback equalizers (DFEs) have been used. DFEs in conjunction with the training data sequence are used to equalize the received data received from these types of channels [37] with this behavior. A minimum mean square error (MMSE) criterion is used to estimate the coefficients of equalizer as well as carrier recovery parameters.

UWA channel is a time variant channel. Least mean squares (LMS) approach can be used to estimate the time delay and equalize such variant channel based on the DFEs equalizer. After demodulation $\mathrm{R}-\mathrm{S}$ decoder is used to recover the decoded image stream, finally decoders of 4 group SPIHT bit strems recover the transmitted image.

Table 1. Underwater acoustic channel profile for simulations.

\begin{tabular}{llllll}
\hline Parameter & value & & & & \\
\cline { 2 - 6 } & 1st path & 2nd path & 3rd path & 4th path & 5th path \\
\hline Amplitude & 0.206 & 0.145 & 0.073 & 0.036 & 0.018 \\
$\begin{array}{l}\text { Relative delay } \\
\text { time (ms) }\end{array}$ & 0 & 0.7 & 2.24 & 4.64 & 9 \\
\hline
\end{tabular}

The Equation (11) is applied to detect the Doppler shift effect and its amplitude in each path. Data symbols are modulated using the BPSK with symbol duration $T=0.4 \mathrm{~ms}$. After demodulation, the $\mathrm{R}-\mathrm{S}$ decoder is used to recover the decoded image stream.

The M-SPHIT encoder output is divided into packets. Each packet has a 2000 bit, which is further divided into 25 blocks with each block to be 80 bits. Each block has 10 symbols with each encoded using one byte. In the simulation results, the encoded Lena image bit-streams are split into 17 packets. M-SPHIT output divides these packets to four separated groups based on its significance. In particular, (1) two of the encoded packets are for the significant bits and they will have the highest entropy, (2) four packets are used to represent the sign bits in the encoded image, (3) nine of the encoded packets are used to represent the set bits, (4) the last two encoded packets are used to represent the refinement bits in the image. At first, the outcome of UEP method are compared with that of the EEP method. For the EEP case, the number of R-S overhead symbols is selected to be six in an encoded packet row. That makes the total number of symbols per packet sent to the modulation to be 400 .

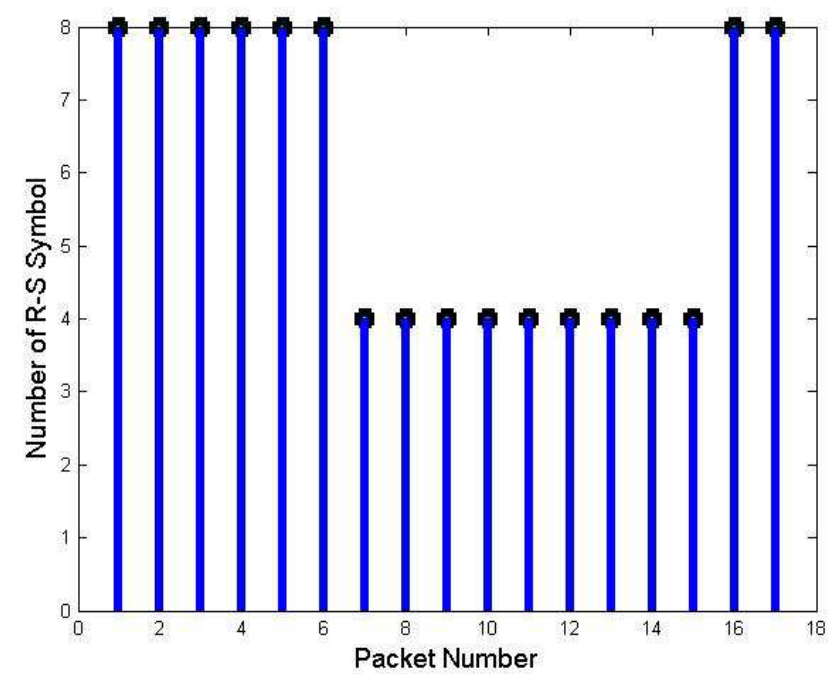

Figure 3. Transmitted "Lena" packets $R-S$ protection levels as determined using the UEP algorithm.

In the case of UEP case, the total number of symbols in all encoded packets in Equation (22) are selected to be no more than that of EEP with R-S symbols equal to 6 symbols, which should be less than channel capacity. For each row of the packet and $\mathrm{R}-\mathrm{S}$ symbols is determined for each encoded 
packet based on the optimization algorithm to minimize the total distortion. As shown in Figure 3, the sign and significance bit-stream blocks are protected by an eight-symbols, considering the low UWA channel signal-to-noise ratio in this case. The set packets groups are protected using four symbols and eight symbols are used to protect the refinement bit-steams.

\subsection{Results}

Image transmission over the underwater channel is simulated under different physical conditions to assess the system performance for both unequal and equal error protection with the $\mathrm{R}-\mathrm{S}$ channel coder. The parameter of wind speed is selected at different values. Each experiment runs for 50 times to collect the average. As shown in Figure 5 and Figure 6, where several variations of images originated from the standard Lena image and dolphin swimming [8, 21], respectively are transmitted through using the proposed underwater communication system. The reconstructed images are obtained at different wind speed levels using the two protection algorithms, unequal and equal error protection, with channel rates distributions for the protection with unequal error protection algorithm case.

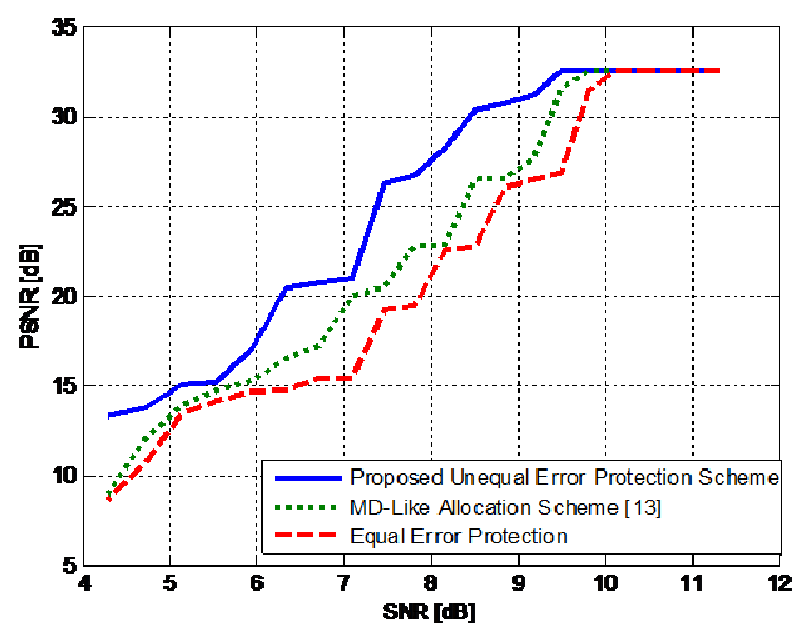

Figure 4. The average PSNR of the received Lena image transmitted over the UWA channel for EEP, MD-Allocation [13] \& proposed UEP channel coding, at channel Doppler shift $=29.6 \mathrm{~Hz}$.

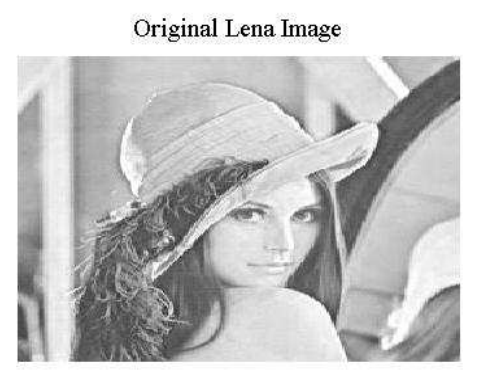

Lena Image received using (UEP), wind $=10$ Knots, $\mathrm{PSNR}=29 \mathrm{~dB}$, total symbol transmitted $=67487$

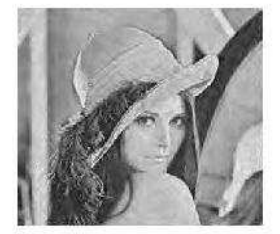

Lena Image received using (UEP), wind $=40$ Knots, $\mathrm{PSNR}=20.4 \mathrm{~dB}$, total symbol transmitted $=67487$

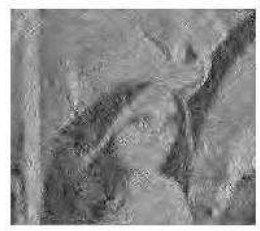

(a)
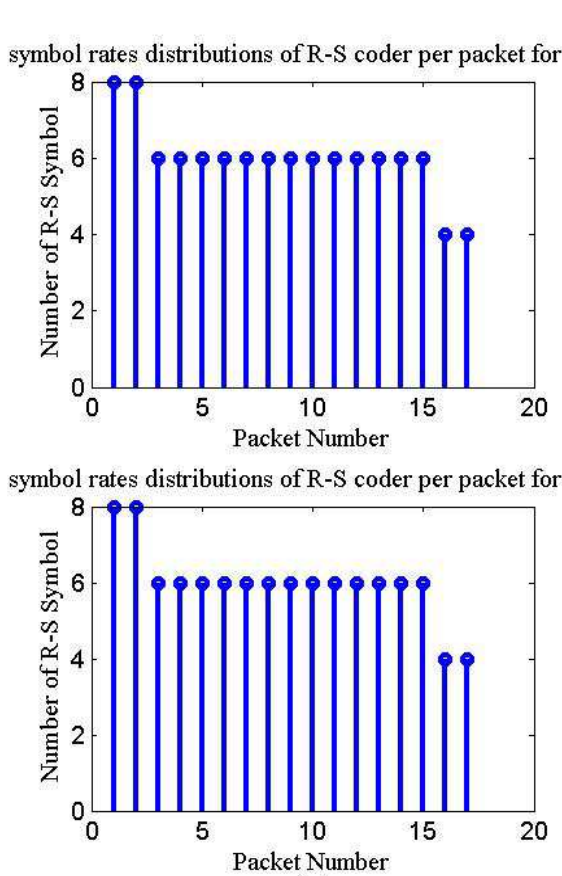

(b)
Lena Image received using (EEP), wind $=10$ Knots, $P S N R=26 \mathrm{~dB}$, total symbol transmitted $=67487$

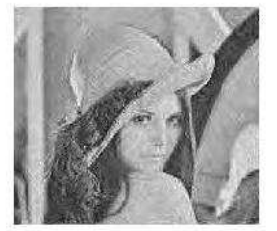

Lena Image received using (EEP), wind $=40$ Knots, PSNR $=17.8 \mathrm{~dB}$, total symbol transmitted $=67487$

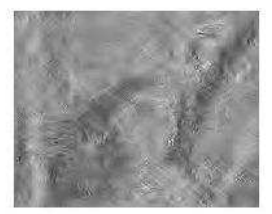

(c)

Figure 5. Reconstructed "Lena" image transmitted over the UWA channel with equal and unequal error protection with channel rate distribution of unequal error protection. 


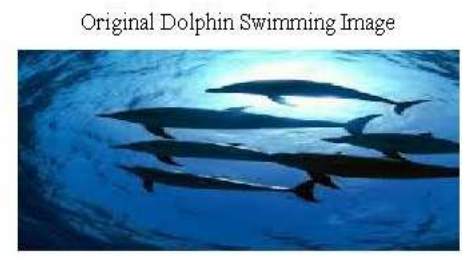

Dolphin Swimming received using (UEP), wind $=10$ Knots, $\mathrm{PSNR}=34.87 \mathrm{~dB}$, total symbol transmitted $=65999$

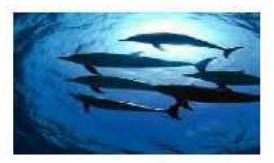

Dolphin Swimming received using (UEP), wind $=25$ Knots, PSNR $=23.3 \mathrm{~dB}$, total symbol transmitted $=67487$

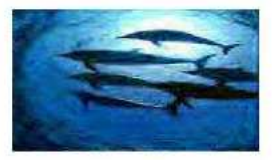

Dolphin Swimming received using (UEP), wind $=40$ Knots, PSNR $=19.9 \mathrm{~dB}$, total symbol transmitted $=66987$

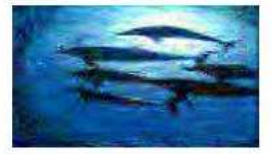

(a)

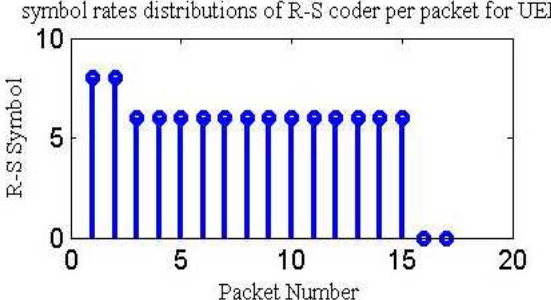

symbol rates distributions of R-S coder per packet for UEP

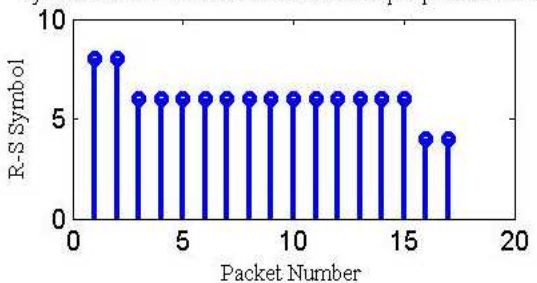

symbol rates distributions of R-S coder per packet for UEP

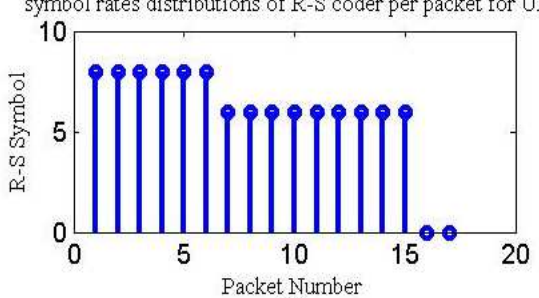

Dolphin Swimming received using (EEP), wind $=10$ Knots, PSNR $=26 \mathrm{~dB}$, total symbol transmitted $=67487$

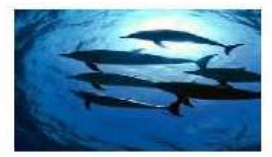

Dolphin Swimming received using (EEP), wind $=25$ Knots, PSNR $=21.4 \mathrm{~dB}$, total symbol transmitted $=67487$

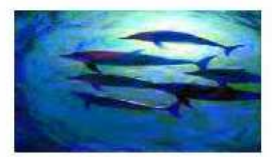

Dolphin Swimming received using (EEP), wind=40 Knots, PSNR $=18.1 \mathrm{~dB}$, total symbol transmitted $=67487$

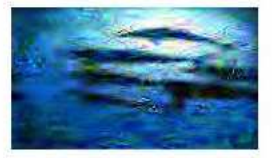

(c)

Figure 6. Reconstructed "Dolphin Swimming" image transmitted over the UWA channel with equal and unequal error protection with channel rate distribution of unequal error protection.

Table 2. Proposed scheme comparison to others.

\begin{tabular}{llll}
\hline Items & Proposed scheme & MD-Like allocation scheme, [11]. & HQAM- approaches [21] \\
\hline Complexity & Complex & Complex & Required \\
CSI (channel state information) & Not required & Required & Not required \\
Feedback from receiver to transmitter & Not required & Valid for any communication & Not required \\
Validity for different communication & Valid for any communication & system & Specific only for HQAM \\
systems & system & 135.6961 & 88.381125 \\
Simulation computation time (seconds) & 151.19477 & & \\
\hline
\end{tabular}

In UEP case, the total number of transmitting symbols ( $R$ in (15)) in the simulation is 67487 . The redundancy symbol number for each encoded group, including the first two packets are significance packet, followed by four sign packets, then nine set packets and two refinement packets in the final, is determined by solving the optimization problem defined in (15). R-S symbol redundancy is shown in Figure 5 (b). In that Figure, the significance packets have eight symbols protection level, the sign and set packets have six symbols protection level, and refinement packets are protected by four symbols. In the simulation of dolphin swimming image, $\mathrm{R}-\mathrm{S}$ rate distribution changes under various channel wind condition, as shown in Figure 6 (b). In particular, at 10 Knot wind the packets of significance type are protected by eight symbols, six symbols are used to protect the sign block and set type of the packets, and the refinement packets are completely unprotected. As the result, the total number of transmitting symbols using UEP is 65999 symbols, which are 1488 symbols less than that obtained using traditional EEP technique.

Figure 4 shows the average PSNR of the received Lena image transmitted over the UWA channel by using the proposed scheme, MD-Like allocation shame [11], and equal forward error correction, respectively, with $\mathrm{R}-\mathrm{S}$ coder all 
coded images transmitted at certain total bit budget.

Due to high UWA channel noise, curves in Figure 4 are not smooth. The unequal error protection encoding method improves the PSNR of the received image. From the simulation results, the proposed UEP method outperforms the conventional EEP system by $3.5 \mathrm{~dB}$ and the system of MD-Like allocation schemes [11] by $2.2 \mathrm{~dB}$ at underwater acoustic channel with Doppler shift equal $29.6 \mathrm{~Hz}$ at fixed total bit budget. The iterative procedure in the proposed system increases the time needed to encode an image, but results in higher PSNR for reconstructing images in low SNR channel which make it more suitable for underwater acoustic channel. Also from the simulation results R-S code is shown to be suitable for an underwater acoustic channel.

In our work in [21], we proposed unequal error protection with only certain communication system Hierarchical Quadrature Amplitude Modulation (HQAM). Table 2 has a conclusion of a comparison between our proposed and other methods.

\section{Conclusion}

In this paper an efficient rate allocation image transmission scheme in underwater acoustic system is discussed. Based on a modified SPIHT coder scheme proposed, a novel image transmission system has been developed. SPIHT coder algorithm has been modified based on the order of significance of the coded bits as well as its contribution in the PSNR of the received image. In particular, four different types of bit-stream are generated by a modified SPIHT coder. The Reed Solomon channel coder is used together with the output of rate allocation vectors to generate the output bit-stream for transmission. While such technique can be considered for generic image transmission issues to achieve reasonable efficiency, an optimization problem is carefully formulated by taking the particular channel feature of underwater acoustic communication channel, such as Doppler shift and probability bit error distribution, into account. As the result, noticeable reduction of the overall distortion over underwater acoustic channel using our proposed technique is observed, together with the improvement in PSNR for the reconstructed image. Simulation experiments are included for illustrative purpose with different image types and different physical parameters such as wind speed. It is shown that the proposed system can effectively reduce the overall distortion in the reconstructed image and improve PSNR with approximately $1.5 \mathrm{~dB}$ in one of the most distorted channels.

\section{References}

[1] I. Iglesias, S. Aijun, J. Garcia-Frias, M. Badiey, and G. R. Arce, "Image transmission over the underwater acoustic channel via compressive sensing," in 45th Annual Conference on Information Sciences and Systems, 2011, pp. 1-6.

[2] D. B. Kilfoyle and A. B. Baggeroer, "The state of the art in underwater acoustic telemetry," IEEE Journal of Oceanic Engineering, vol. 25, pp. 4-27, 2000.
[3] J. Rice, B. Creber, C. Fletcher, P. Baxley, K. Rogers, K. McDonald, et al., "Evolution of seaweb underwater acoustic networking," in Oceans, 2000, pp. 2007-2017.

[4] M. Stojanovic, "Underwater acoustic communication," Wiley Encyclopedia of Electrical and Electronics Engineering, 1999.

[5] C. Murphy and H. Singh, "Wavelet compression with set partitioning for low bandwidth telemetry from AUVs," in Proceedings of the Fifth ACM International Workshop on UnderWater Networks, 2010, pp. 1-8.

[6] C. Pelekanakis, M. Stojanovic, and L. Freitag, "High rate acoustic link for underwater video transmission," in OCEANS, 2003, pp. 1091-1097.

[7] A. Said and W. A. Pearlman, "A new, fast, and efficient image codec based on set partitioning in hierarchical trees," IEEE Transactions on Circuits and systems for video technology, vol. 6, pp. 243-250, 1996.

[8] H. Esmaiel and D. Jiang, "SPIHT coded image transmission over underwater acoustic channel with unequal error protection using HQAM," in IEEE International Conference on Information Science and Technology (ICIST), 2013, pp. 1365-1371.

[9] W. C. Cox, J. A. Simpson, C. P. Domizioli, J. F. Muth, and B. L. Hughes, "An underwater optical communication system implementing Reed-Solomon channel coding," in OCEANS, 2008, pp. 1-6.

[10] P. A. Chou, A. E. Mohr, A. Wang, and S. Mehrotra, "Error control for receiver-driven layered multicast of audio and video," IEEE Transactions on Multimedia, vol. 3, pp. 108-122, 2001.

[11] B. Tomasi, L. Toni, P. Casari, J. Preisig, and M. Zorzi, "A study on the SPIHT image coding technique for underwater acoustic communications," in Proceedings of the Sixth ACM International Workshop on Underwater Networks, 2011, p. 9.

[12] M. A. Kader, F. Ghani, and R. B. Ahmed, "Unequal Error Protection for SPIHT Coded Image Transmission over Erroneous Wireless Channels," Asian Transactions on Fundamentals of Electronics, Communication \& Multimedia, vol. 1, pp. 1-6, 2011.

[13] U. S. Mohammed and H. Hamada, "An efficient rate allocation scheme with selective weighted function for optimum peak-to-average power ratio for transmission of image streams over OFDM channels," International Journal of Video \& Image Processing \& Network Security, vol. 9, 2009.

[14] U. S. Mohammed and H. Hamada, "Image transmission over OFDM channel with rate allocation scheme and minimum peak-toaverage power ratio," Journal of Telecommunication, vol. 2, p. 70:78, 2010.

[15] Y. Sun, R.-m. Li, and X.-1. Cao, "Image compression method of terrain based on Antonini wavelet transform," in IEEE International Geoscience and Remote Sensing Symposium, 2005, pp. 692-695.

[16] E. Christophe and W. A. Pearlman, "Three-dimensional SPIHT coding of volume images with random access and resolution scalability," Journal on Image and Video Processing, vol. 2008, pp. 1-13, 2008.

[17] Z. Lu, D. Y. Kim, and W. A. Pearlman, "Wavelet compression of ECG signals by the set partitioning in hierarchical trees algorithm," IEEE Transactions on Biomedical Engineering, vol. 47, pp. 849-856, 2000. 
[18] J. Preisig, "Acoustic propagation considerations for underwater acoustic communications network development," $A C M$ SIGMOBILE Mobile Computing and Communications Review, vol. 11, pp. 2-10, 2007.

[19] K. Prasanth, "Modelling and Simulation of an Underwater Acoustic Communication Channel," Master, Electronic Engineering University of applied sciences, Bremen, Germany, 2004.

[20] H. Esmaiel and D. Jiang, "Review Article: Multicarrier Communication for Underwater Acoustic Channel," International Journal of Communications, Network and System Sciences, vol. 6, pp. 361-376, 2013.

[21] H. Esmaiel and D. Jiang, "Image transmission over underwater acoustic environment using OFDM technique with HQAM mapper," in IEEE International Conference on Information Science and Technology (ICIST), 2013, pp. 1596-1601.

[22] Y. Chen, X. Xu, L. Zhang, and Z. Zou, "Design and Application of dynamic coding in shallow water acoustic communications," in OCEANS, 2012, pp. 1-6.

[23] A. Sehgal, I. Tumar, and J. Schönwälder, "AquaTools: An Underwater Acoustic Networking Simulation Toolkit," Oceans, pp. 1-6, 2010 .

[24] A. Fish, A. Sayeed, S. Gurevich, R. Hadani, and O. Schwartz, "Delay-Doppler channel estimation with almost linear complexity: To Solomon Golomb for the occasion of his 80 birthday mazel tov," in IEEE International Symposium on Information Theory Proceedings, 2012, pp. 2386-2390.

[25] J. Trubuil and T. Chonavel, "Accurate Doppler estimation for underwater acoustic communications," in OCEANS, 2012, pp. $1-5$.

[26] A. Katariya, A. Arya, and K. Minda, "Coded under Water Acoustic Communication (UWA) with Cryptography," in International Conference on Computational Intelligence and Communication Networks, 2010, pp. 493-497.

[27] S. Sandberg and N. Von Deetzen, "Design of bandwidth-efficient unequal error protection LDPC codes,"
IEEE Transactions on Communications, vol. 58, pp. 802-811, 2010.

[28] A. Albanese, J. Blomer, J. Edmonds, M. Luby, and M. Sudan, "Priority encoding transmission," IEEE Transactions on Information Theory, vol. 42, pp. 1737-1744, 1996.

[29] R. Puri and K. Ramchandran, "Multiple description source coding using forward error correction codes," in Conference Record of the Thirty-Third Asilomar Conference on Signals, Systems, and Computers, 1999, pp. 342-346.

[30] A. Goalic, J. Trubuil, C. Laot, and N. Beuzelin, "Underwater acoustic communication using Reed Solomon Block Turbo Codes channel coding to transmit images and speech," in OCEANS, 2010, pp. 1-6.

[31] J. P. Odenwalder, "Error control coding handbook," DTIC Document1976.

[32] M. Chitre, "A high-frequency warm shallow water acoustic communications channel model and measurements," The Journal of the Acoustical Society of America, vol. 122, p. 2580, 2007.

[33] M. Stojanovic, J. Catipovic, and J. G. Proakis, "Adaptive multichannel combining and equalization for underwater acoustic communications," The Journal of the Acoustical Society of America, vol. 94, p. 1621, 1993.

[34] M. A. Chitre, J. R. Potter, and S.-H. Ong, "Optimal and near-optimal signal detection in snapping shrimp dominated ambient noise," IEEE Journal of Oceanic Engineering, vol. 31, pp. 497-503, 2006.

[35] J. S. Panaro, F. R. Lopes, L. M. Barreira, and F. E. Souza, "Underwater Acoustic Noise Model for Shallow Water Communications," in Simpósio Brasileiro de Telecomunicações, 2012, pp. 1-4.

[36] C. T. Kelley, Iterative methods for optimization vol. 18: Society for Industrial and Applied Mathematics, 1987.

[37] M. Stojanovic, "Recent advances in high-speed underwater acoustic communications," IEEE Journal of Oceanic Engineering, vol. 21, pp. 125-136, 1996. 